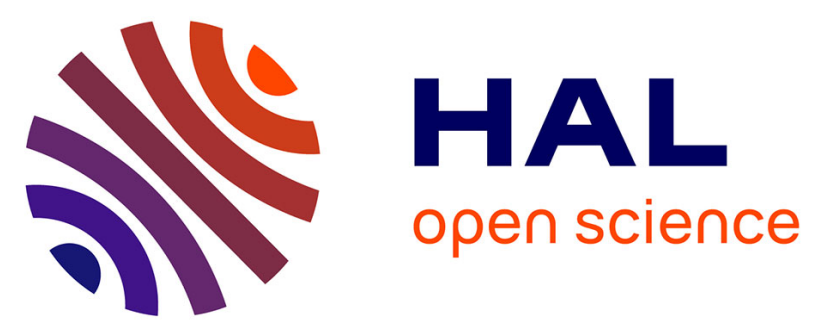

\title{
Assessment of risk factors for occurrence or worsening of acetabular fracture following percutaneous cementoplasty of acetabulum malignancies
}

\author{
Marie-Charlotte Hesler, Xavier Buy, Vittorio Catena, Véronique Brouste, \\ Michèle Kind, Jean Palussière, Amandine Crombé
}

\section{To cite this version:}

Marie-Charlotte Hesler, Xavier Buy, Vittorio Catena, Véronique Brouste, Michèle Kind, et al.. Assessment of risk factors for occurrence or worsening of acetabular fracture following percutaneous cementoplasty of acetabulum malignancies. European Journal of Radiology, 2019, 120, pp.108694 -. 10.1016/j.ejrad.2019.108694 . hal-03488784

\section{HAL Id: hal-03488784 \\ https://hal.science/hal-03488784}

Submitted on 21 Dec 2021

HAL is a multi-disciplinary open access archive for the deposit and dissemination of scientific research documents, whether they are published or not. The documents may come from teaching and research institutions in France or abroad, or from public or private research centers.
L'archive ouverte pluridisciplinaire HAL, est destinée au dépôt et à la diffusion de documents scientifiques de niveau recherche, publiés ou non, émanant des établissements d'enseignement et de recherche français ou étrangers, des laboratoires publics ou privés.

\section{()ㅜ(1)}

Distributed under a Creative Commons Attribution - NonCommerciall 4.0 International 


\section{TITLE}

\section{Assessment of Risk Factors for Occurrence or Worsening of Acetabular Fracture Following Percutaneous Cementoplasty of Acetabulum Malignancies}

Marie-Charlotte Hesler ${ }^{1}$, Xavier Buy ${ }^{1}$, Vittorio Catena ${ }^{1}$, Véronique Brouste ${ }^{2}$, Michèle Kind ${ }^{1}$, Jean Palussière ${ }^{1}$, Amandine Crombé $^{1,3}$

1. Department of Diagnostic and Interventional Radiology, Institut Bergonié, Regional Comprehensive Cancer Center, F-33076, Bordeaux, France

2. Department of Clinical Epidemiology Research, Institut Bergonié, F-33076, Bordeaux, France.

3. University of Bordeaux, IMB, UMR CNRS 5251, INRIA Project team Monc F-33400 Talence, France

\section{Corresponding author:}

Dr Amandine Crombé, MD, MS

Email: a.crombe@bordeaux.unicancer.fr

Tel: +33(0) 556333333

Fax: +33(0) 556333330

Address: Department of Diagnostic and Interventional Radiology, Institut Bergonié, Regional Comprehensive Cancer Center, 229 cours de l'Argonne, F-33000, Bordeaux, France 


\title{
TITLE
}

\section{Assessment of Risk Factors for Occurrence or Worsening of Acetabular Fracture Following Percutaneous Cementoplasty of Acetabulum Malignancies}

\begin{abstract}
Purpose: to evaluate the clinical, radiological and periprocedural features associated with the occurrence or worsening of acetabular fracture (OWAF) following percutaneous cementoplasty of the acetabulum (PCA) in cancer patients.
\end{abstract}

Method: all patients who underwent PCA in our comprehensive cancer center for an acetabular metastasis between January 2008 and December 2015 were included. Clinical features, characteristics of the metastasis on computed tomography (CT-scan) (location [roof, quadrilateral surface, anterior and posterior columns], number of locations, matrix, extra/intra-articular fractures, extra-osseous or subchondral extensions) and of the procedure (extra- or intra-articular cement leakage (IACL), percentage of filling of each location, complications) were reported as well as prior, concomitant or post-PCA treatments. The endpoint was OWAF on CT-scan during post-PCA follow-up. Log-rank tests and Cox models were used to identify prognostic factors.

Results: 140 PCA were identified in 129 patients (11 bilateral procedures, median age: 66.6). Eighteen $(18 / 140,12.9 \%)$ had an initial articular fracture. IACL was seen in 12/140 (8.6\%) PCA. The only feature associated with IACL was a pre-existing articular fracture $(\mathrm{p}=0.009)$. Of the 111 patients with imaging follow-ups, 18 (16.2\%) showed OWAF. In multivariate analysis, the presence of cement filling (even partial) of all acetabular metastatic locations was the only feature predictive of OWAF-free survival (hazard ratio=3.8, $\mathrm{p}=0.031$ ). 
Conclusions: Injecting cement in all areas affected by acetabular metastases could prevent OWAF. Because survival following PCA is not negligible, completing an insufficient first PCA could help preserve patients' quality of life.

\section{Keywords}

Neoplasm Metastasis; Bone Neoplasms; Acetabulum; Cementoplasty; Radiology, Interventional

\section{Abbreviations}

IACL: intra-articular cement leakage

C195\%: 95\% confidence interval

CT: computed tomography

HR: hazard ratio

OR: odds ratio

OS: overall survival

OWAF: occurrence or worsening of an acetabular fracture

PCA: percutaneous cementoplasty of the acetabulum

RFA: radiofrequency ablation

SD: standard deviation 


\section{Introduction}

Bone metastases occur in about 30-70\% of cancer patients [1]. Among these, acetabular metastases are often painful and can lead to pathological hip fractures resulting in serious loss of autonomy. Radiotherapy alone can help reducing pain but does not strengthen this osteopenic area nor does it help healing fractures [2-4]. Furthermore, 20-40\% of patients do not respond to radiotherapy [5]. Cancer patients with acetabular metastases are usually poor candidates for surgical reconstructive hip procedures. This surgery is complex and may be discussed in the case of slow evolving limited metastatic diseases. Hence, minimally invasive image-guided percutaneous injections of cement have been suggested to consolidate pathologic acetabulum, potentially combined with a local treatment of the metastasis i.e. radiofrequency ablation (RFA), cryoablation, microwave ablation or arterial embolization [68]. The first studies about percutaneous cementoplasty of the acetabulum (PCA) reported early pain relief, hip stabilization and improved walking abilities in most patients [9-11]. The main complications of the procedure were extra- or intra-articular cement leakages (IACL), which may irritate and/or compress nerves, thereby worsening hip pains, and migrate into vascular structures leading to embolism. However, the mid- to long-term effects of PCA on acetabular pathological fractures and patients' survival have been poorly evaluated. Herein, based on our 7 year-long experience in a comprehensive cancer center, we aimed at investigating: (i) the risk factors of periprocedural cement leakage, (ii) the risk factors of occurrence of new pathological acetabular fractures (or worsening of pre-existing fractures) following a PCA and (iii) the patients' outcome after PCA.

\section{Materials and Methods}

\subsection{Study Population}


Our institutional review board approved this retrospective single-center study. All consecutive adult patients were included as they underwent a PCA in our department between January 2008 and December 2015 because of a painful bone metastasis of the acetabulum or an acetabular metastasis at risk of fracturing. Metastases were diagnosed by MRI or computed tomography (CT). All the procedures were validated by a multidisciplinary board that included oncologists, interventional radiologists, radiotherapists and orthopaedic surgeons. The following clinical data were reported: gender, age, histotype and prior local treatment at least 3 months before PCA (diphosphonates, radiotherapy, thermal-ablation and arterial embolization). Figure 1 shows the flow-chart of the study, which consisted in 3 steps: (i) assessment of risk factors for IACL, (ii) assessment of risk factors for occurrence or the worsening of an acetabular fracture (OWAF) and (iii) assessment of the prognostic value of OWAF in term of patients' overall survival in the whole population as well as in the most frequent histological type of the series.

\subsection{Pre-PCA radiological evaluation}

Two radiologists $(\mathrm{MCH}, \mathrm{XB})$ analyzed together and in consensus the imaging that was acquired just before the PCA and reported on: (1) the metastasis matrix (categorized as lytic, sclerotic, mixed), (2) the location(s) of the metastasis (categorized as roof, quadrilateral surface, anterior and/or posterior columns), (3) the number of locations (from 1 to 4 ), the presence of an extension involving (4) the subchondral bone (categorized as absent, minor if $<25 \%$ of articular surface, major if $\geq 25 \%$ of articular surface, or non-analyzable in case of hip replacement) or (5) the extra-osseous soft-tissues.

\subsection{PCA procedure}


The procedures were performed by 3 senior interventional radiologists with more than 10 years of experience in cementoplasty (JP, XB, VC) under general anaesthesia, cardiovascular monitoring, periprocedural antibioprophylaxis and extensive local sterility. The patients were placed in prone, supine or lateral position depending on the lesion location. The procedures were guided by CT (lightSpeed 16, GE Healthcare) or by cone-beam CT equipped with XPerCT and X-PerGuide ${ }^{\circledR}$ tools (Philips Healthcare). Bone-bevelled needles (11-13Gx10$15 \mathrm{~cm}$ long, Cook) were used to access the acetabular metastasis. Once correctly positioned, a maximal amount of polymethyl-methacrylate radio-opaque bone cement was injected under continuous imaging guidance. If there was a risk of intra- or extra-articular cement leakage, the injection was stopped. Two cements were used either with low viscosity (Opacity+, Teknimed) or with high viscosity (F20, Teknimed) depending on the risk of cement leakage. Depending on the extension of the acetabular metastasis, one or two needles were used. In this latter case, the cement filling was performed sequentially and not simultaneously. However, the first needle was always left with the stylet until the end of the second filling.

Regarding patients with a concomitant local treatment, RFA was carried out just before cementoplasty with a probe inserted into the metastasis and performed with one straight impedance-based electrode (Cool-tip, Covidien). The ablative protocol lasted 8-12 min based on the impedance constantly showed by the system. At the end of the ablation, the system automatically showed the temperature reached in the treated area; a temperature $>60^{\circ} \mathrm{C}$ was considered lethal. For concomitant cryoablation, the procedure consisted of alternate cycles of freezing and thawing obtained by rapid decompression respectively of argon and helium gases into the distal tip of the probes (IceRod, IceSphere, IceSeed, Galil Medical). While performing freezing cycles, CT or CBCT scans were periodically obtained every 3-5 min to monitor the progressive growth of the hypodense ice-ball (i.e. ablation area). 


\subsection{Periprocedural and post-procedure evaluations}

Periprocedural evaluation consisted in assessing: (1) the occurrence of intra- and or extraarticular cement leakage, (2) concomitant local treatments (radiotherapy within 3 months before or after PCA, thermal-ablation, embolization), (3) cement filling of each metastasis location (using a 3-point scale, categorized as $0 \%,<50 \%$ or $\geq 50 \%$ ) to estimate the overall filling rate of the procedure (at least one location without any cement filling vs. at least one location with filling $<50 \%$ vs. all locations filled $\geq 50 \%$ ).

Two radiologists $(\mathrm{MCH}, \mathrm{XB})$ read together and in consensus all the follow-up CTs that were performed after the PCA. They noted the occurrence or the worsening of an acetabular fracture and consequently if another local post-PCA treatment was performed (additional cementoplasty, radiation therapy, thermal-ablation, embolization, diphosphonates). This enabled us to calculate the survival from initial PCA to OWAF in months (OWAF-free survival). Patients without a CT performed more than 30 days after the procedure were removed from this survival analysis. Radiation therapy, whenever it was performed, consisted in 20 Gy in 5 fractions over 1 week. A worsening of a pre-existing fracture was defined as a widening $>2 \mathrm{~mm}$ of the fracture or the occurrence of a new fracture line from the pre-existing fracture.

\subsection{Statistical analysis}

Associations between categorical or ordinal variables and IACL were assessed with Chisquare tests. The prognostic value of the clinical, pre- and per-PCA features in terms of OWAF-free survival was investigated. Survival curves were generated using the KaplanMeier method. The impact of the variables found to be associated with a p-value $<0.200$ in univariate analysis was evaluated in multivariate Cox regression. The following co-variables were used for adjustment as potential confounding data: age $(<$ or $\geq$ median age $)$, gender, 
histotype, pre-PCA local treatment (at least one vs. none), post-PCA local treatment (at least one vs. none), and post-PCA additional cementoplasty. Results of the modeling are given as hazard ratio (HR) with 95\% confidence interval (CI95\%). Finally, the impact of OWAF on overall survival (OS) following PCA was estimated using log-rank test. Patients without event (i.e. censored patient) during a follow-up of less than 6 months were removed from the OS analysis. All tests were two-tailed. Patients with a missing value were removed from the multivariable analyses. Statistical analyses were done using SPSS (version 21.0, IBM). A pvalue $<0.05$ was deemed significant.

\section{Results}

\subsection{Patients}

In total, 140 PCA carried out on 129 patients (80 women, median age: 66 year-old, range: $37-$ 90). Eleven patients had a bilateral PCA, which was performed at the same time for 9 of them. Table 1 summarizes the initial demographic data of the whole population. The most common histotype was breast cancer $(52 / 129,40.3 \%)$.

Table 2 shows the radiological features of the acetabulum metastases. Most were lytic lesions $(84 / 140,60 \%)$ with a major subchondral extension $(61 / 140,43.6 \%)$. Roof location was the most frequent $(128 / 140,87.1 \%)$ but patients generally presented with several locations in the acetabulum. A unique location was seen in only $25 / 140$ (17.9\%) of the procedures. Eighteen acetabulums showed an articular fracture (18/140, 12.9\%).

\subsection{Assessment of pre- and per-PCA features at risk of IACL}

Table 3 summarizes the characteristics of the procedures. IACL was observed during 12/140 procedures $(8.6 \%)$ and extra-articular cement leakage in 58/140 procedures (41.4\%). Neither the clinical characteristics of the patients, nor the radiological features of the metastases were 
associated with IACL, except for the presence of an articular fracture $(\mathrm{p}=0.009)$ (Table 3). The odds ratio for periprocedural IACL in case of articular acetabular fracture was 6.3 $(\mathrm{CI} 95 \%=[1.8-22.8])$. Figure 2 shows a case of IACL favored by pathological intra-articular acetabular fracture.

\subsection{Outcome}

Of the 140 PCA, 111 (79.3\%) were included in the survival analysis. Twenty-nine PCAs did not have a sufficient radiological follow-up at our institution and were punctually addressed to our center for the PCA. The median overall survival of the series was 20.7 months $(C 195 \%=[13.9-27.6])$. Eighteen PCAs $(18 / 111,16.2 \%)$ demonstrated OWAF during the follow-up (4 worsening and 14 new fractures). From the initial PCA to the diagnosis of OWAF, 32/111 (28.8\%) procedures underwent at least one additional PCA and 39/107 (36.4\%) procedures required at least another post-PCA treatment (4 patients with missing data).

Table 4 summarizes the survival analysis in the whole study population (supplementary table provides the predictive value of initial clinical features). In the univariate analysis, 3 features had a p-value < 0.200: a major subchondral bone extension $(\mathrm{p}=0.164)$, a concomitant treatment $(\mathrm{p}=0.109)$ and the absence of cement filling of one of the locations reached by the acetabular metastasis $(\mathrm{p}=0.187)$. After adjustment with potential confounders, this last variable was the only independent predictor of OWAF $(\mathrm{HR}=3.8, \mathrm{CI} 95 \%=[1.1-13.2]$, $\mathrm{p}=0.031$ ). In the whole population, the 3-year OWAF-free survival probabilities was $86 \%$ $(\mathrm{CI} 95 \%=[74.2-97.8])$ in patient with all tumor locations filled, no matter the amount of cement, while it was $73 \%(\mathrm{CI} 95 \%=[61-85])$ in patients without any filling of at least one location. Once the OWAF was diagnosed, the therapeutic management consisted in 2 full hip replacements, 9 additional PCAs including 2 with concomitant local radiotherapies. 


\subsection{Prognostic value of $O W A F$}

Regarding the prognostic value of OWAF in the whole population study ( $\mathrm{n}=106$ with available radiological follow-up and overall survival information), 53/88 (59.6\%) patients without OWAF died while 14/18 (77.8\%) patients with OWAF did. Patients with OWAF had a significantly lower OS compared to patients without (median OS $=15.2$ months, $\mathrm{CI} 95 \%=[9.2-21.2]$ versus 28.3 months, CI95\% $=[17.7-38.9]$, respectively, $\mathrm{p}=0.028)($ Fig. 3). Notably, in the population of breast cancer patients, i.e. the most common histological types of the series ( $\mathrm{n}=46$ patients with available radiological follow-up and overall survival data), 7/7 (100\%) patients with OWAF died while 21/39 (53.8\%) patients without OWAF did. In this subgroup, OWAF remained a prognostic factor for overall survival (median OS $=21.9$ months $\mathrm{CI} 95 \%=[8.6-35.2]$ in patients with OWAF versus 39.4 months $\mathrm{CI} 95 \%=[27.9-60.9]$ in patients without OWAF, $\mathrm{p}=0.010$ ). Figures 4 and 5 show two opposite examples of patients with and without OWAF and their outcome.

\section{Discussion}

With the improvements in systemic therapeutics for metastatic cancer patients, overall survival has increased. Besides the global control of the disease, a new challenge for these patients is to preserve a good quality of life. Acetabular metastases are paradigmatic of this issue: they are not life threatening but patients are at high risk of losing their autonomy. In this study, the OS following a PCA was significant. Indeed, about $60 \%$ and $40 \%$ of patients were still alive respectively one and two years after PCA. Moreover, we found that an incomplete procedure (i.e. the lack of cement filling of at least one of the acetabular locations of the metastasis) was an independent prognostic factor for an OWAF after the initial PCA. 
One of the most frequent reasons for stopping a PCA is the occurrence of an IACL during the procedure. Our results indicate that a pre-existing articular fracture was its main risk factor.

Herein, PCA appears to be a safe procedure. Only 12/140 procedures demonstrated an IACL. No severe per-PCA complications were reported. These results concur with the literature. Cement leaks in the hip joint never exceeded 1-3 cases per prior study and no serious adverse event ever occurred during PCA [9-13]. Weill et al. hypothesized that there was a logical association between articular fracture and IACL [10]. In their series, the 3 patients with IACL had an articular fracture on the CT performed just before.

The sole periprocedural prognostic factor of OWAF in our multivariate analysis was the lack of filling of at least one acetabular area reached by the metastasis. On the contrary, none of the radiological characteristics of the acetabular malignancy was predictive of OWAF, that-isto-say the distribution of the metastasis, the tumor matrix, the presence of subchondral and extra-osseous extensions, pre-PCA fractures or IACL. In a recent study focusing on PCA combined with cryoablation, Kurup et al. highlighted an association between surgery or radiation therapy performed before PCA and new fractures after PCA [8]. Herein, this association was not retained but the differences between procedures (systematically combined with cryotherapy in their study), the population sizes and the statistical designs (associations in their case, and survival analysis taking into account the delay following the procedure) may explain the difference in the results. In our study, only one patient with concomitant cementoplasty and thermal ablation had a prior radiation therapy, hence, the lack of observations and events limited the possibility to perform the same analysis as Kurup et al. $[8]$.

Complete cement filling of the whole metastasis was rarely achieved. In fact, many patients had metastases that spread in different locations of the acetabulum. The main location was 
generally filled at $\geq 50 \%$ and the other locations, less at risk of fracturing according to the operator, were partly filled. Sixty-two patients had no cement filling on one of the metastatic locations. These results suggest that a stabilization effect and a reduction of the risks of fracture do not require an entire filling of the lesions but at least a partial filling of all anatomical locations in the acetabulum invaded by the tumor. This partial but exhaustive filling would significantly strengthen the hip joint by increasing resistance to compression and by reducing microtrauma. Hence, we believe that interventional radiologists should not hesitate to re-iterate a PCA shortly after an incomplete first PCA, before the occurrence of new pain and fractures, in order to complete the filling of all the pathological locations of the acetabulum. It should be noted that some patients from our series required additional local treatment in order to prevent the occurrence of new fractures. Thirty-two patients had at least one additional cementoplasty and 39 patients had additional local treatments during the follow-up (before being censored or before OWAF). Consequently, we added the covariables 'post-PCA cementoplasty' and 'post-PCA local treatment' in the multivariate survival analyses to limit the confusion bias.

As previously noticed, a pre-existing articular fracture was not a predictive factor of a new fracture. This could mean that PCA, possibly in combination with other local treatments, should not be restricted to acetabular metastases at risk of fracturing but could be prescribed for patients with actual non-displaced articular fractures too [8].

The median OS of our series was about 21 months, which is not negligible for metastatic cancer patients and highlight that some cancers could almost be considered as chronic diseases. The high proportion of patients with breast cancer could explain this and that is why we focused on this subgroup to confirm the relationship between OWAF and OS. Ensuring a good quality of life, which relies on pain relief and normal mobility to perform daily activities, is one of the major objectives of the multidisciplinary board in charge of patients. 
Herein, we showed that the occurrence or aggravation of an acetabular fracture following PCA was a prognostic factor for OS in the whole population, but also in a more homogeneous subgroup of metastatic breast cancer patients. Even if these results are retrospective and extracted from a univariate analysis, they show that a good local control of the metastatic disease, particularly when it involves weight-bearing body areas crucial to the patient's autonomy, may influence the patients' outcome. Furthermore, to our knowledge, our study provides one of the largest and longest follow-ups after PCA - prior studies did not exceed a follow-up of 6 months - and this relationship between local stabilization after PCA and OS has never been identified. Our series also illustrate that PCA can easily be integrated with radiotherapy and does not preclude further PCAs or hip replacements.

The main limit of our study is its retrospective nature. Indeed, questionnaires enabling the quantification of pain and function before and after PCA were not available although they could have provided original information regarding the duration of pain relief following PCA and its impact on daily activities. It should be noted that prior studies have shown pain relief up to 2 years $[14,15]$. Recently, Moser et al. showed that cementoplasty of pelvic bone metastases significantly reduced the pain assessed with visual analog scale [16]. Some technical variables were not analyzed in our study, such as the amount of cement, the duration of the procedure and the number of needles and positionings, as well as variables characterizing the fracture such as its exact location, the gap between the 2 pieces, the delay from diagnosis to treatment, an underlying osteopenia and/or sarcopenia. Interestingly, there was no correlation with the filling percentage in the study by Moser et al. but the authors stressed the added value of cement injection optimization through the use several needles and the simultaneous injection of cement [16]. Indeed, simultaneous injection may improve the coalescence of the cement, hence providing a more compact and robust block of cement. On 
the contrary, sequential injection may be at higher risk of cement leakage along the track of the first inserted needle. However, it should be noted that this risk is considerably decreased when the interventional radiologist leaves the first needle with its stylet until the complete end of the filling, which was always the case at our institution [16]. Consequently, herein, we do not believe that a sequential filling could have led to an increased rate of cement leakage. Furthermore, the cement filling could have been improved with balloon-assisted osteoplasty/kyphoplasty, as proposed by Kurup et al. [17]. This technic could help to enhance the distribution and coalescence of cement.

In our study, the technique was not standardized since some patients had a concomitant treatment including thermal ablations. To limit the bias induced by associated treatments (before, periprocedural, post-procedure), we introduced these confounding covariables in the multivariate analysis. It should be kept in mind that our first aim was not to compare the techniques and therapeutic strategies but to identify features associated with OWAF. Furthermore, the follow-up by CT-scan was not standardized and 29 patients had to be removed from the OWAF-free survival analysis. Some patients were closely followed-up notably those included in clinical trials who underwent a CT-scan about every 2 months and an additional CT-scan in case of abnormal pain, while other patients did not have any CTscans following PCA. There were 2 reasons for the missing information: an early death and the fact that some patients were treated outside of our center and only referred to us for a PCA. For these last patients, we did not have the knowledge of prior or post-PCA treatment. Consequently, the delay from PCA to OWAF and the prevalence of OWAF may have been underestimated. Moreover, we did not include the general and local response status of the patients among the confounding covariables. It could be hypothesized that patients with a progressive disease or a locally progressive disease would be at higher risk of unfavorable events such as a pathological fracture or even death. Furthermore, the evolution of the bone 
metastases under treatment could be another factor to consider because some may consolidate. Another limit is the method of quantification of cement filling. We suggested a 3-point scale however a more precise quantification should be developed. Finally, some recent techniques were not represented in our study, in particular the screw fixation under imaging-guidance that may improve hip stabilization and reduce the risk of fracture[7, 18, 19]. Our group is prospectively evaluating the optimal indications for adding screw fixation to cement injection or patients with pelvic bone metastases. Moreover, screw fixation may be appropriate during additional PCAs to counterbalance the lack of cohesion of cement blocks that were injected during different sessions.

In conclusion, this study reflects the 7-year-long experience of a comprehensive cancer center regarding PCA and provides original insights about the radiological practices for acetabulum malignancies. We showed that IACL were significantly associated with pre-existing articular fractures and that the cement filling, even incomplete, of all acetabulum areas significantly decreased the risk of OWAF. Because OWAF may be associated with worse OS, we would recommend an interventionist therapeutic attitude with additional PCA or a new procedure such as screw fixation for patients with incomplete cement filling before the occurrence of new pain and/or fractures.

\section{References}

[1] Guzik G. Treatment of metastatic lesions localized in the acetabulum. J Orthop Surg. (2016) 11:54.

[2] Poulsen HS, Nielsen OS, Klee M, Rørth M. Palliative irradiation of bone metastases. Cancer Treat Rev. 16 (1989) 41-48. 
[3] Jeremic B, Shibamoto Y, Acimovic L, et al. A randomized trial of three single-dose radiation therapy regimens in the treatment of metastatic bone pain. Int $\mathrm{J}$ Radiat Oncol Biol Phys. 42 (1998) 161-167

[4] Gaze MN, Kelly CG, Kerr GR, et al. Pain relief and quality of life following radiotherapy for bone metastases: a randomised trial of two fractionation schedules. Radiother Oncol J Eur Soc Ther Radiol Oncol. 45 (1997) 109-116

[5] Rich SE, Chow R, Raman S, et al. Update of the systematic review of palliative radiation therapy fractionation for bone metastases. Radiother Oncol J Eur Soc Ther Radiol Oncol. 126 (2018) 547-557.

[6] Lane MD, Le HBQ, Lee S, et al. Combination radiofrequency ablation and cementoplasty for palliative treatment of painful neoplastic bone metastasis: experience with 53 treated lesions in 36 patients. Skeletal Radiol. 40 (2011) 25-32.

[7] Pusceddu C, Sotgia B, Fele RM, et al. Combined Microwave Ablation and Cementoplasty in Patients with Painful Bone Metastases at High Risk of Fracture. Cardiovasc Intervent Radiol. 39 (2016) 74-80.

[8] Kurup AN, Schmit GD, Morris JM, et al. Avoiding Complications in Bone and Soft Tissue Ablation. Cardiovasc Intervent Radiol. 40 (2017) 166-176.

[9] Cotten A, Deprez X, Migaud H, et al. Malignant acetabular osteolyses: percutaneous injection of acrylic bone cement. Radiology. 197 (1995) 307-310.

[10] Weill A, Kobaiter H, Chiras J. Acetabulum malignancies: technique and impact on pain of percutaneous injection of acrylic surgical cement. Eur Radiol. 8 (1998) 123-129.

[11] Maccauro G, Liuzza F, Scaramuzzo L, et al. Percutaneous acetabuloplasty for metastatic acetabular lesions. BMC Musculoskelet Disord. (2008) 9:66.

[12] Gupta AC, Hirsch JA, Chaudhry ZA, et al. Evaluating the safety and effectiveness of percutaneous acetabuloplasty. J Neurointerventional Surg. 4 (2012) 134-138. 
[13] Wallace AN, Huang AJ, Vaswani D, et al. Combination acetabular radiofrequency ablation and cementoplasty using a navigational radiofrequency ablation device and ultrahigh viscosity cement: technical note. Skeletal Radiol. 45 (2016) 401-405.

[14] Kelekis A, Cornelis FH, Tutton S, Filippiadis D. Metastatic Osseous Pain Control: Bone Ablation and Cementoplasty. Semin Interv Radiol. 34 (2017) 328-336.

[15] Kelekis A, Lovblad KO, Mehdizade A, et al. Pelvic osteoplasty in osteolytic metastases: technical approach under fluoroscopic guidance and early clinical results. J Vasc Interv Radiol JVIR. 16 (2005) 81-88.

[16] Moser TP, Onate M, Achour K, Freire V. Cementoplasty of pelvic bone metastases: systematic assessment of lesion filling and other factors that could affect the clinical outcomes. Skeletal Radiol. (2019) Feb 2. doi: 10.1007/s00256-019-3156-0.

[17] Kurup AN, Morris JM, GD Smith, et al. Balloon-Assisted Osteoplasty of Periacetabular Tumors following Percutaneous Cryoablation. J Vasc Interv Radiol JVIR. 26 (2015) 588-594. [18] Laredo J-D, Mosseri J, Nizard R. Percutaneous Nailing and Cementoplasty for Palliative Management of Supra-Acetabular Iliac Wing Metastases: A Case Report. JBJS Case Connect. (2017) 7:e46.

[19] Buy X, Catena V, Roubaud G, et al. Image-Guided Bone Consolidation in Oncology. Semin Interv Radiol. 35 (2018) 221-228. 


\section{Figure and Tables}

Table 1. Initial clinical characteristics of the population.

Table 2. Characteristics of the acetabulum metastases.

Table 3. Assessment of features associated with the occurrence of intra-articular leakage during the percutaneous acetabular cementoplasty procedure.

Table 4. Prognostic value of the radiological and periprocedural features to predict occurrence or worsening of acetabular fracture following a percutaneous cementoplasty of the acetabulum.

Figure 1. Flow-chart of the study.

Abbreviations: OS: overall survival, OWAF: occurrence or worsening of an acetabular fracture following percutaneous cementoplasty of the acetabulum, PCA: percutaneous cementoplasty of the acetabulum. Patients were removed from the part 3 of the study if their follow-up was of less than 6 months without event (i.e. censored patient within the $1^{\text {st }}$ sixth months)

Figure 2. Intra-articular cement leakage during a percutaneous cementoplasty of the acetabulum (PCA). A 44 year-old woman presented with a lytic and sclerotic metastasis of the acetabulum secondary to metastatic breast cancer. The lesion involved the roof, the anterior and posterior columns and was complicated by an articular fracture as seen on coronal and sagittal computed tomography reformations (A, black arrow heads). The lesion was first treated by radiation therapy. A PCA was secondarily performed under fluoroscopy guidance 
with two $13 \mathrm{G}$ needles $(\mathbf{B})$. During the procedure, an intra-articular cement leakage was seen (C, white arrow) and confirmed on the post-PCA computed tomography, performed 1 month later (D).

Figure 3. Survival analysis. Kaplan-Meier curves for overall survival depending on occurrence or worsening of acetabular fracture since percutaneous cementoplasty of the acetabulum in the whole population study (A) and in the subgroup of patients with metastatic breast cancer $(\mathbf{B}) . *$ : $p<0.05$

Figure 4. New fractures and worsening of pre-existing fractures following percutaneous cementoplasty of the acetabulum (PCA). A 82 year-old patient with metastatic breast cancer underwent a PCA following a local radiation therapy for a lytic metastasis of the left acetabulum involving the quadrilateral surface, the roof, the anterior and the posterior columns). The procedure had to be stopped because of an intra-articular cement leakage. Hence, even if the quadrilateral surface was correctly filled with cement, the filling of the posterior column was incomplete $(<50 \%)$ and no cement was injected in the anterior column and in the roof (A - upper image: sagittal view of the CT-scan before PCA; lower image: sagittal view of the CT-scan post-PCA, white arrow). (B) On the axial view of the CT-scan performed 67 days after PCA, there was a widening (i.e. worsening) of the fracture of the posterior column (dashed white arrow) and two new fractures were diagnosed (white arrow heads). The patient died of her disease 17 months later.

Figure 5. Favorable outcome following a percutaneous cementoplasty of the acetabulum (PCA). A 62 year-old woman with metastatic breast cancer presented with a bone metastasis of the left acetabulum involving all the locations with a major subchondral extension (A, 
coronal view of CT-scan). Because of pain and high risk of fracture, a PCA with concomitant local radiation therapy were indicated. There was no complication during the procedure. PostPCA computed tomography showed a good diffusion of the cement in all areas $(\mathbf{B}$, axial and sagittal reformation). During the post-PCA follow-up, the patient underwent the positioning of a screw in the ilio-pubic branch with a cement injection. The last computed tomography did not demonstrate additional fracture 18 months later and the patient is still alive 2 years later. 


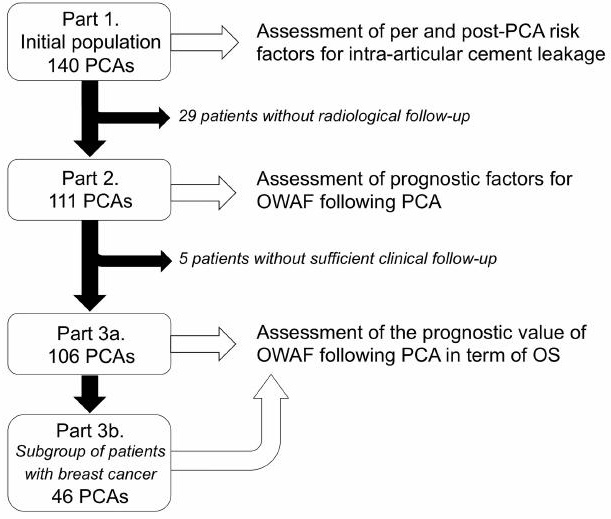




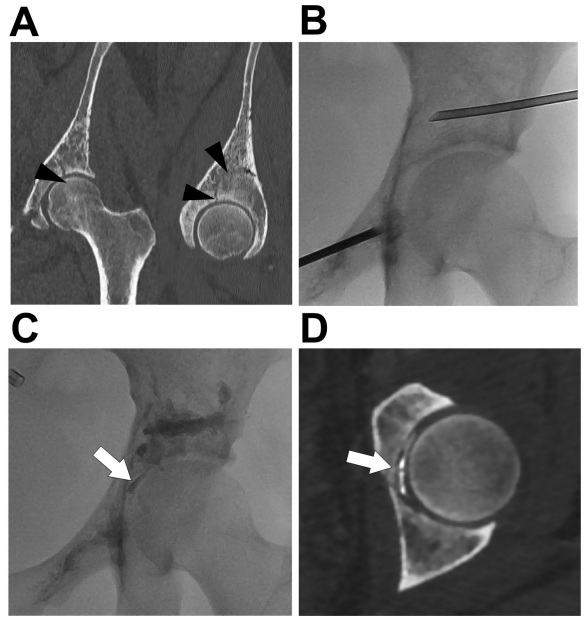



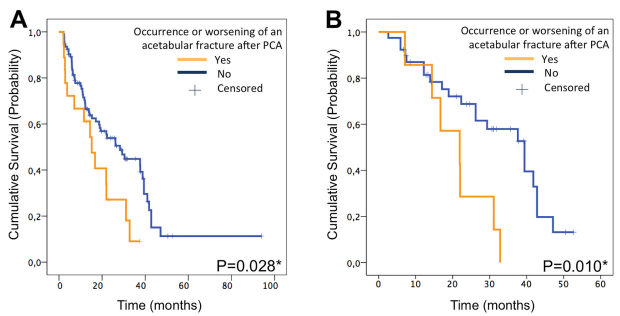
$\mathbf{A}$
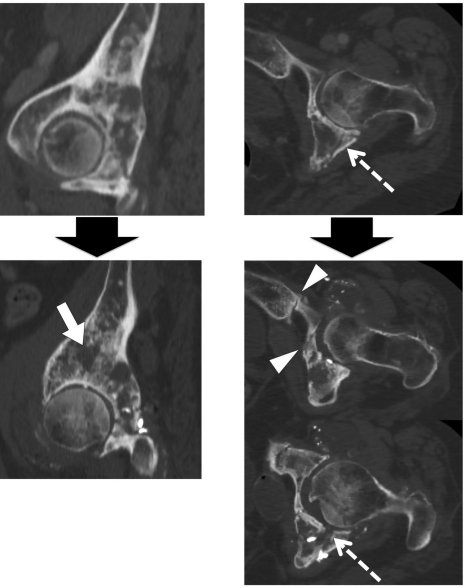

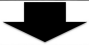

$\sum_{-1}$

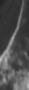

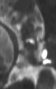

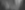


$\mathbf{A}$

B

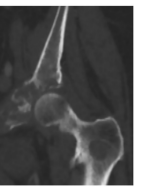

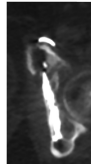

is
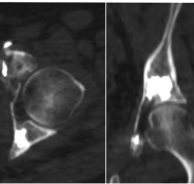
Table 1. Initial clinical characteristics of the population

\begin{tabular}{lc}
\hline Characteristics & Patients \\
\hline Age (mean +/- sd) & $66.2+/-11.1$ \\
median, range & $66(37-90)$ \\
Gender & \\
Male & $49 / 129(38)$ \\
Female & $80 / 129(62)$ \\
Initial cancer site & \\
Breast & $52 / 129(40.3)$ \\
Kidney & $14 / 129(10.9)$ \\
Prostate & $13 / 129(10.8)$ \\
Lung & $12 / 129(9.3)$ \\
Urinary tract & $10 / 129(7.8)$ \\
Myeloma & $8 / 129(6.2)$ \\
Thyroid & $6 / 129(4.7)$ \\
Soft-tissue sarcoma & $5 / 129(4)$ \\
Other ${ }^{1}$ & $9 / 129(7)$ \\
Bilateral percutaneous acetabuloplasty & \\
Unilateral & $118 / 129(91.5)$ \\
Bilateral ${ }^{2}$ & $11 / 129(8.5)$ \\
Prior local treatment & \\
Biphosphonates & \\
Radiation therapy & $26 / 126(20.6)$ \\
Thermal ablation & $29 / 133(21.8)$ \\
Embolization & $5 / 137(3.6)$ \\
Articular fractures of the acetabulum & $2 / 137(1.5)$ \\
Absent & \\
Present & $122 / 140(87.1)$ \\
\hline NOT Data are number & $18 / 140(12.9)$ \\
\hline
\end{tabular}

NOTE: Data are number of patients with percentage in parentheses, except for age. Abbreviations : sd : standard deviation.

${ }^{1}$ Other tumors are: hepatocellular carcinoma $(n=2)$, colon cancer $(n=2)$, oesophagal cancer $(n=1)$, Head and neck cancer $(n=1)$, adenocarcinoma of unknown origin $(n=3)$.

${ }^{2}$ The contralateral percutaneous acetabuloplasty was performed in the same time for 9 patients and 4.3 months later for the 2 other patients.

${ }^{3}$ Prior local treatment of the acetabulum were performed at least 3 months prior to the current procedure. Information about the prior local treatments were missing in the medical records for 14 acetabulums regarding biphosphonates, 7 regarding radiation therapy, 3 regarding thermal ablation and 3 regarding tumor embolization. 
Table 2. Characteristics of the acetabulum metastases

\begin{tabular}{lc}
\hline Characteristics & Procedures $^{\S}$ \\
\hline Tumor matrix & \\
Lytic & $84 / 140(60)$ \\
Osteosclerotic & $6 / 140(4.3)$ \\
$\quad$ Mixed & $50 / 140(35.7)$ \\
Soft-tissue extension & \\
Absent & $99 / 140(70.7)$ \\
Present & $41 / 140(29.3)$ \\
Subchondral bone extension & \\
Absent & $29 / 140(20.7)$ \\
Minor & $43 / 140(30.7)$ \\
Major & $61 / 140(43.6)$ \\
Total hip replacement ${ }^{1}$ & $7 / 140(5)$ \\
Articular fracture & \\
Absent & $122 / 140(87.1)$ \\
Present & $18 / 140(12.9)$ \\
Acetabular metastasis location & \\
Roof & $128 / 140(91.4)$ \\
Anterior column & $84 / 140(60)$ \\
Posterior column & $97 / 140(69.3)$ \\
Quadrilateral surface & $86 / 140(61.4)$ \\
Number of locations ${ }^{2}$ & \\
1 of 4 & $25 / 140(17.9)$ \\
2 of 4 & $32 / 140(22.9)$ \\
3 of 4 & $26 / 140(18.6)$ \\
4 of 4 & $57 / 140(40.7)$ \\
\hline
\end{tabular}

NOTE. Data are number of patients with percentage in parenthese.

$\S$ : A total of 140 percutaneous acetabular cementoplasty were performed in 129 patients, 11 of them having a bilateral procedure.

${ }^{1}$ Subchondral bone extension was not analyzable in 7 patients because of total hip replacement

${ }^{2}$ The number of locations corresponds to the number of anatomical location of the acetabulum affected by the bone metastasis among: roof, anterior column, posterior column and quadrilateral surface. 
Table 3. Assessment of features associated with the occurrence of intra-articular leakage during the percutaneous acetabular cementoplasty procedure.

\begin{tabular}{|c|c|c|c|}
\hline Characteristics & $\begin{array}{c}\text { No intra-articular } \\
\text { leakage }\end{array}$ & $\begin{array}{l}\text { Intra-articular } \\
\text { leakage }\end{array}$ & p-value \\
\hline \multicolumn{4}{|l|}{ Gender } \\
\hline Male & $50 / 128(39.1)$ & $3 / 12(25)$ & 0.530 \\
\hline Female & $78 / 128(60.9)$ & $9 / 12(75)$ & \\
\hline \multicolumn{4}{|l|}{ Age $^{1}$} \\
\hline$\leq 66$ years-old & $64 / 128(50)$ & $5 / 12(41.7)$ & 0.580 \\
\hline$>66$ yeas-old & $64 / 128(50)$ & $7 / 12(58.3)$ & \\
\hline \multicolumn{4}{|l|}{ Initial cancer site } \\
\hline Breast & $50 / 128(39.1)$ & $7 / 12(58.3)$ & 0.604 \\
\hline Kidney & $13 / 128(10,1)$ & $1 / 12(8.3)$ & \\
\hline Prostate & $13 / 128(10.1)$ & $1 / 12(8.3)$ & \\
\hline Lung & $14 / 128(10.9)$ & $0 / 12(0)$ & \\
\hline Urinary tract & $12 / 128(9.4)$ & $0 / 12(0)$ & \\
\hline Others & $26 / 128(20.3)$ & $3 / 12(25)$ & \\
\hline \multicolumn{4}{|c|}{ Prior radiation therapy } \\
\hline No & $94 / 122(77)$ & 8/11 (72.7) & 0.720 \\
\hline Yes & $28 / 122(23)$ & 3/11 (27.3) & \\
\hline \multicolumn{4}{|c|}{ Prior thermoablation } \\
\hline No & $121 / 125(96.8)$ & $11 / 12(91.7)$ & 0.370 \\
\hline Yes & $4 / 125(3.2)$ & $1 / 12(8.3)$ & \\
\hline \multicolumn{4}{|c|}{ Prior diphosphonates } \\
\hline No & $89 / 114(78.1)$ & $11 / 12(91.7)$ & 0.240 \\
\hline Yes & $25 / 114(21.9)$ & $1 / 12(8.3)$ & \\
\hline \multicolumn{4}{|l|}{ Prior embolization } \\
\hline No & $124 / 125(99.2)$ & $11 / 12(91.7)$ & - \\
\hline Yes & $1 / 125(0.8)$ & $1 / 12(8.3)$ & \\
\hline \multicolumn{4}{|c|}{ Subchondral bone extension ${ }^{2}$} \\
\hline No & 28/121 (23.1) & $1 / 12(8.3)$ & 0.277 \\
\hline Yes, minor & $40 / 121(33.1)$ & $3 / 12(25)$ & \\
\hline Yes, major & $53 / 121(43.8)$ & $8 / 12(66.7)$ & \\
\hline \multicolumn{4}{|c|}{ Extra-osseous bone extension } \\
\hline No & $92 / 128(71.9)$ & $7 / 12(58.3)$ & 0.330 \\
\hline Yes & $36 / 128(28.1)$ & $5 / 12(41.7)$ & \\
\hline \multicolumn{4}{|c|}{$\mathrm{Nb}$ of locations in the acetabulum } \\
\hline$\leq 2$ & $55 / 128(43)$ & $2 / 12(16.7)$ & 0.120 \\
\hline$>2$ & $73 / 128(57)$ & $10 / 12(83.3)$ & \\
\hline \multicolumn{4}{|c|}{ Osteosclerotic metastasis ${ }^{3}$} \\
\hline No & $76 / 128(59.4)$ & $8 / 12(66.7)$ & 0.760 \\
\hline Yes & $52 / 128(40.6)$ & $4 / 12(33.3)$ & \\
\hline \multicolumn{4}{|l|}{ Lytic metastasis ${ }^{3}$} \\
\hline No & $6 / 128(4,7)$ & $0 / 12(0)$ & 1.000 \\
\hline Yes & $122 / 128(95.3)$ & $12 / 12(100)$ & \\
\hline \multicolumn{4}{|l|}{ Articular fracture } \\
\hline Absent & $115 / 128(89.9)$ & $7 / 12(58.3)$ & $0.009 *$ \\
\hline Present & $13 / 128(10.1)$ & $5 / 12(41.7)$ & \\
\hline
\end{tabular}

NOTE. Data are number of procedures for which the variable was analysable, with percentage in parentheses.

${ }^{1}$ The cut-off for dichotomisation of age was defined as the median of the population of the study.

${ }^{2}$ For subchondral bone extension, patients with total hip replacement were removed.

${ }^{3}$ Mixed lesions with both sclerotic and lytic lesions were included in both categories, i.e. presence of lytic metastasis and presence of sclerotic metastasis.

$*: \mathrm{p}<0.05$. 
Table 4. Prognostic value of the radiological and per-procedure features to predict occurrence or worsening of acetabular fracture following a percutaneous cementoplasty of the acetabulum.

\begin{tabular}{|c|c|c|c|c|c|}
\hline \multirow{2}{*}{ Characteristics } & \multirow{2}{*}{$\begin{array}{c}\text { No. Of } \\
\text { procedures }\end{array}$} & \multirow{2}{*}{$\begin{array}{l}\text { No. Of } \\
\text { events }\end{array}$} & \multirow{2}{*}{$\begin{array}{c}\text { log-rank } \\
\text { p-value }\end{array}$} & \multicolumn{2}{|c|}{ Multivariate analysis $^{1}$} \\
\hline & & & & HR (CI95\%) & p-value \\
\hline \multicolumn{6}{|l|}{ Metastasis involving the roof } \\
\hline No & 9 & 2 & & & \\
\hline Yes & 102 & 16 & 0.771 & - & - \\
\hline \multicolumn{6}{|c|}{ Metastasis involving the anterior column } \\
\hline No & 44 & 9 & & & \\
\hline Yes & 67 & 9 & 0.225 & - & - \\
\hline \multicolumn{6}{|c|}{ Metastasis involving the posterior column } \\
\hline No & 36 & 6 & & & \\
\hline Yes & 75 & 12 & 0.731 & & \\
\hline \multicolumn{6}{|c|}{ Metastasis involving the quadrilateral surface } \\
\hline No & 42 & 6 & & & \\
\hline Yes & 69 & 12 & 0.788 & - & - \\
\hline \multicolumn{6}{|l|}{ Number of locations } \\
\hline 1 location & 18 & 3 & & & \\
\hline 2 locations & 28 & 4 & & & \\
\hline 3 locations & 24 & 6 & & & \\
\hline 4 locations & 41 & 5 & 0.531 & - & - \\
\hline \multicolumn{6}{|l|}{ No. of locations in the acetabulum } \\
\hline$\leq 2$ & 45 & 7 & & & \\
\hline$>2$ & 66 & 11 & 0.985 & - & - \\
\hline \multicolumn{6}{|l|}{ Subchondral bone extension } \\
\hline No or minor & 59 & 8 & & & \\
\hline Major & 46 & 10 & 0.164 & $1.7(0.5-5.2)$ & 0.375 \\
\hline \multicolumn{6}{|l|}{ Subchondral bone extension } \\
\hline No & 23 & 3 & & & \\
\hline Minor or Major & 82 & 15 & 0.397 & - & - \\
\hline \multicolumn{6}{|l|}{ Extra-osseous bone extension } \\
\hline No & 78 & 13 & & & \\
\hline Yes & 33 & 5 & 0.963 & - & - \\
\hline Purely lytic lesion & & & & & \\
\hline No & 48 & 7 & & & \\
\hline Yes & 63 & 11 & 0.732 & - & - \\
\hline Purely osteosclerotic lesion & & & & & \\
\hline No & 102 & 16 & & & \\
\hline Yes & 9 & 2 & 0.535 & - & - \\
\hline Mixed lesion & & & & & \\
\hline No & 68 & 11 & & & \\
\hline Yes & 43 & 7 & 0.997 & - & - \\
\hline Articular fracture & & & & & \\
\hline Absent & 96 & 15 & & & \\
\hline Present & 15 & 3 & 0.616 & - & - \\
\hline Intra-articular cement leakage & & & & & \\
\hline Absent & 100 & 15 & & & \\
\hline Present & 11 & 3 & 0.364 & - & - \\
\hline Concomitant radiation therapy & & & & & \\
\hline No & 83 & 12 & & & \\
\hline Yes & 25 & 6 & 0.185 & - & - \\
\hline Concomitant thermal ablation & & & & & \\
\hline No & 102 & 17 & & & \\
\hline Yes & 9 & 1 & 0.344 & - & - \\
\hline Concomitant embolization & & & & & \\
\hline No & 93 & 16 & & & \\
\hline Yes & 15 & 2 & 0.803 & - & - \\
\hline At least one concomitant treatme & & & & & \\
\hline No & 81 & 11 & & & \\
\hline Yes & 27 & 7 & 0.109 & $2.1(0.7-6.6)$ & 0.200 \\
\hline Filling of the lesions & & & & & \\
\hline Non-filling of one area & 62 & 13 & & & \\
\hline Filling of all areas $(<50 \%)$ & 46 & 5 & & & \\
\hline Filling of all areas $(\geq 50 \%)$ & 3 & 0 & 0.363 & - & - \\
\hline Filling of the lesions (dichotomiz & & & & & \\
\hline Filling of all areas $(<$ or $\geq 50 \%)$ & 49 & 5 & & & \\
\hline Non-filling of one area & 62 & 13 & 0.187 & $3.8(1.1-13.2)$ & $0.031 *$ \\
\hline
\end{tabular}


NOTE. No.: number; HR: hazard ratio; CI95\%: 95\% confidence interval The minimal follow-up by CT-scan was 1 month. Patients who died before OWAF were censored. Comparisons were assessed by the Log-rank test.

1/ The variables with a p-value $<0.200$ at univariate analysis were entered in a multivariate Cox model in which the following co-variables were included for adjustment and to avoid confusion bias: gender (male vs. female), age ( $<$ or $\geq 66$ years-olds i.e. median agE), histotype (breast vs. lung vs. prostate vs. urinary tract vs. others), prePCA local treatment (at least one vs. none), post-PCA local treatments (at least one vs. none), post-PCA cementoplasty (at least one vs. none). Procedures with missing value(s) were excluded from the analysis. In total, 100 procedures were analysed.

$*: \mathrm{p}<0.05$ 